\title{
Interoperability Testing Suite for C2X Communication Components
}

\author{
Fabian de Ponte Müller ${ }^{1}$, Juan María Reveriego Sierra ${ }^{2}$, Bernhard Kloiber ${ }^{1}$, \\ Matthias Röckl ${ }^{1}$, and Thomas Strang ${ }^{1}$ \\ 1 Institute of Communications and Navigation, \\ German Aerospace Center, Munich, \\ Germany \\ 2 University of Málaga, \\ Málaga, Spain
}

\begin{abstract}
This paper presents a collection procedures to perform interoperability tests of $\mathrm{C} 2 \mathrm{X}$ communication equipment. Following a cross layer approach, interoperability test purposes on radiocommunication equipment, networking layer algorithms, C2X applications and use cases are analyzed. Along with this analysis the main conclusions gathered from the execution of the proposed test cases on a concrete C2X prototype system will be presented.
\end{abstract}

Keywords: interoperability testing, C2X communication, geographical routing, physical layer, medium access, use case, test case.

\section{Introduction}

The European commission has distributed in the last years research funding for European field operational tests for Intelligent Transportation Systems (ITS) in order to accelerate the development of C2X prototypes across Europe. Most likely these prototypes include hardware and software components developed by different manufacturers. However, experiences have shown that during the integration phase on the field large effort has to be invested in solving interoperability issues between devices, specially in case of devices brought together by different partners. A solution to overcome this issue is conducting proper defined test cases to verify the correct interoperability between devices.

Many ITS applications rely on direct communication between vehicles (V2V) or vehicles and infrastructure (V2I). While numerous communication methods for ITS systems are defined [2], including 3G, Bluetooth, IrDA, 802.11a/b/g or $802.11 \mathrm{p}$, it is the latter, which is likely to be in charge of direct vehicle to vehicle communication. $802.11 \mathrm{p}$ is rather a young standard with practically no final products available on the market. This work will concentrate on prototypes following IEEE 802.11p or its European equivalent ITS-G5.

Fig. 1] shows a usual representation of the protocol stack of an ITS system. Interoperability issues do not appear only on the lowest communication layers. 
Misconfiguration or a faulty implementation of a standard can lead to interoperability problems on networking, facility or application layer. For this reason a layered approach has been followed throughout this work. First the lowest communication layers are tested, then moving upwards through the communication stack, the networking layer and the $\mathrm{C} 2 \mathrm{X}$ applications are regarded. This way, interoperability failures from lower layers can be discarded when observing high level failures. In order to compose this suite of test cases, on every layer the corresponding standard or specification has been taken and analyzed for interoperability issues.

The test cases analyzed in the scope of this work have been carried out on an ITS implementation developed within the European project PreDrive C2X and which follows the common European architecture defined by COMeSafety [2] The employed communication devices are prototypes from manufacturers such as NEC1 1 , DENSO 2 and Delph 3 . These communication devices contain the lowest communication layers, namely the physical layer, the data link layer and the networking layer. The networking layer within the project is based on the GeoNet specification [5] for geographical addressing and routing, likely to be standardized for ITS at European level by ETSI. Two implementations of this standard, by NEC and Hitachi, have been tested. The facility layer, which provides services for the running applications, and the applications itself were developed by different partners and are running on a open source OSGI implementation on an automotive PC. The applications that have been tested for interoperability are road works warning, car breakdown warning, green light optimal speed advisory, in-vehicle signage and electronic emergency brakelight.

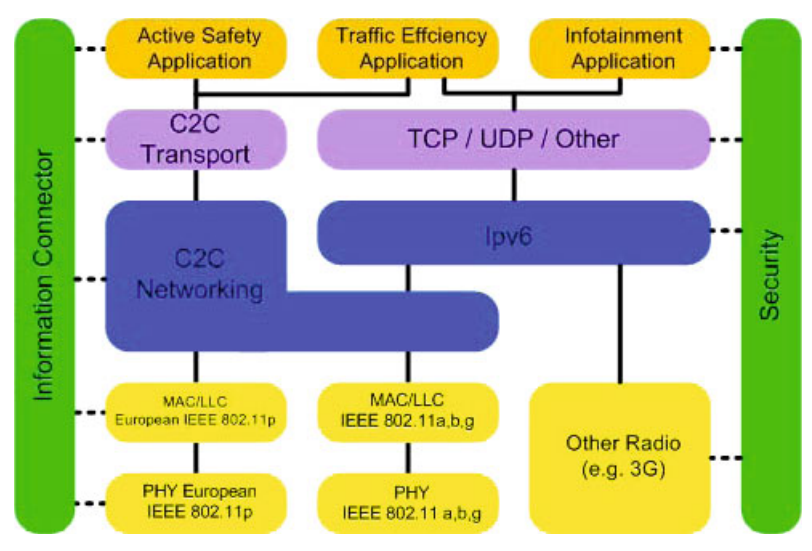

Fig. 1. Reference protocol stack for the common European architecture 2

\footnotetext{
${ }_{1}^{1}$ NEC Europe Ltd.

${ }^{2}$ DENSO International America, Inc.

${ }^{3}$ Delphi Delco Electronics Europe GmbH.

${ }^{4}$ Hitachi Europe SAS.
} 


\section{Test Cases}

The performance verification of a single equipment against its specification is known as conformance testing. Conformance testing is accomplished using a reference system at standardized interfaces, which are normally not accessible to the user or developer. In this sense we expect our systems under test (SUT) to have been proven for conformance by the system manufacturer in advance. Interoperability testing, on the other hand, proves the end-to-end functionality of the whole set of equipments in its conglomerate [3].

In interoperability testing there is always one item which is the subject of the test, the equipment under test (EUT) and a qualified equipment (QE) that has already been proven to interoperate. Due to the lack of a QE which all other EUT may be tested against, the test purposes presented here follow a test configuration where two or more EUT are tested against each other. When a EUT proves to pass a test it can be stated to be a QE to perform tests on further devices. Fig. 2

In the next sections different test cases on different communication layers will be identified. The test set-up and the test procedure will be commented and a set of verification parameters will be specified. Finally, the test verdicts for the given prototypes will be presented for each layer.

\subsection{Physical Layer Testing}

Physical layer testing is the first step in verifying the correct interoperability of $\mathrm{C} 2 \mathrm{X}$ radiocommunication devices. Once direct communication between two devices is proven, further tests on networking or application layer can be performed without the concern of a failure at the lowest layers. The physical layer used in European systems for car-to-car and car-to-infrastructure communication is defined in ETSI ITS-G5 standard (ES 202-663 9]), which is a slightly modified version of IEEE's 802.11p amendment for ITS systems. As the given devices under test work in the frequency band from $5855 \mathrm{M} \mathrm{Hz}$ to $5905 \mathrm{M} \mathrm{Hz}$ the requirements for G5A and G5B will be examined next.

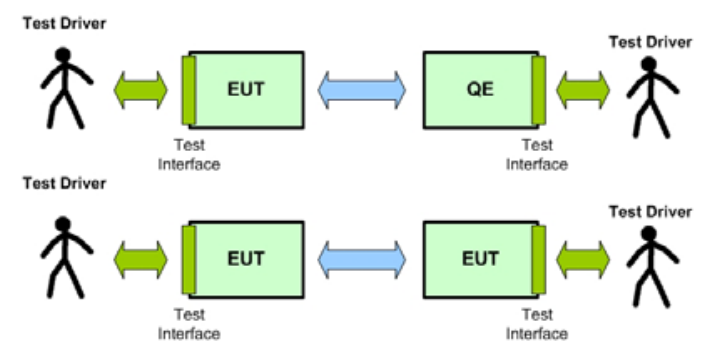

Fig. 2. Illustration of main interoperability testing concepts 
- Frequency Allocation: ITS devices should be able to tune to any of the five $10 M h z$ channels in the G5A and G5B band, namely channels G5CC (control channel) and G5SC1 to G5SC4 (service channel), which correspond to IEEE channels 172-180.

- Channel Bandwidth: According to the standard the supported channel bandwidth should be $10 \mathrm{MHz}$.

- Dual receiver: The standard requires ITS devices to listen to the control channel while not transmitting. Practically, this results in a dual receiver concept, as radio devices are not able to switch without some delay.

- Data rate: The transmitted data rate is changed by using different modulation scheme/coding rate pairs. Default values for the data rate are $6 \mathrm{Mbps}$ on channels G5CC and G5SC1 and 12Mbps on the rest.

Test parameters related to RF output power, transmit power control, power spectral density or unwanted emissions fall out of the scope of interoperability testing and are part of previous conformance tests [8]. All the aforementioned communication units feature WLAN miniPCI cards based on Atheros chipsets, which prove to be flexible enough to be tuned with the help of a suited driver (madwifi, ath5k, etc.) to meet the European standard. Practically all ITS prototypes, however, can modify their transmission parameters prior utilization by means of some graphical configuration tool or a mere command line application, making the parameter setting transparent to the user/tester. For instance, the communication units used during this work have configuration files and configuration scripts where the radio interface can be set appropriately. According to this, different test cases have been identified. The first three test cases should be performed indoors, placing the devices at an appropriate distance from each other (i.e. far field). This way, the tests are independent on the output power, the receiver sensitivity or the channel characteristics.

- Transmission channel: Sequentially, a pair of communication devices should be tuned to a common channel and the ability to communicate should be verified. 0\% PER (Packet Error Rate) is expected to PASS the test.

- Bit rate: This test case proves the ability of devices to communicate at different bit rates. The communication bit rate is given by the transmitter, which defines it in the header of the PHY frame. The header itself is coded always at $3 \mathrm{Mbps}$. Although the default is $6 \mathrm{Mbps}$ (12Mbps on G5SC2) other possible bitrates are 3, 4.5, 9, 18, 24 and 27Mbps. Communication using these values should be verified requiring in each case a $0 \%$ PER to PASS the test.

- Dual receiver: ETSI ITS-G5 standard requires de facto that every ITS device features two radio interfaces, one of them tuned on channel G5CC. The test requires the setup of three devices, one radio transmitting on the G5CC, a 
second radio transmitting on one of the $\mathrm{G} 5 \mathrm{SC}$ and a third radio as EUT listening on both channels. Under this configuration simultaneous reception on a service and the control channel should be verified, requiring $0 \% \mathrm{PER}$ to PASS the test.

- Transmission range: This test is important to be able to determine the achievable communication range with given radio devices, $\mathrm{RF}$ cables and antennas. Obviously the possible communication range depends on several environmental factors, which moreover are difficult to quantify. Obstacles causing shadowing, diffraction or attenuation, surfaces that cause multipath propagation, line of sight (LOS)/non line of sight (NLOS) propagation conditions, transmitter/receiver movement or in-band interferers and noise are examples of phenomena that have a negative impact on the transmission range. Unlike the tests mentioned so far, this one is carried out outdoors using two devices in different vehicles and mounting the antennas following the manufacturer's advisories (cabling, ground plane, etc.). The test should be carried out under controlled and reproducible conditions with regard to LOS, reflections, etc.. The devices are progressively separated from each other until packet loss increases over 0\%. Having a GNSS receiver attached to the communication device, the distance between transmitter and receiver can be evaluated. Although the minimum transmission range is not standardized by ETSI, the experiences gathered during test trials in the last years yield a minimum range of $500 \mathrm{~m}$ under LOS conditions and maximum transmit power. According to C2C-Communication Consortium [1], safety applications require a direct communication range of at least $300 \mathrm{~m}$.

In order to perform these tests, two communication devices from the same or different vendors are needed. On one of the communication devices an application for generating traffic is needed, while the other device should have an application to receive traffic, log it, calculate the packet error rate (PER) and verify its completeness. Test traffic contains an identifier, a timestamp, a sequence number and, if available, position information. As the test setup includes only one sending device, the isolation of the MAC layer algorithm is assured, i.e. the MAC layer will always sense an idle carrier. Table 1 summarizes the test results for the three radiocommunication devices under test.

One of the ITS devices did not have two radio interfaces installed, thus, being unable to receive on the control channel and on a service channel at the same time. The transmission range test revealed a poor communication range

Table 1. Test results for physical layer interoperability tests

\begin{tabular}{|l||c|c|c|}
\hline Test Case & Radio A & Radio B & Radio C \\
\hline Transmission channel & PASS & PASS & PASS \\
Bit rate & PASS & PASS & PASS \\
Dual receiver & PASS & PASS & FAIL \\
Transmission range & FAIL & FAIL & FAIL \\
\hline
\end{tabular}


of $100 \mathrm{~m}$. This test was performed outdoors under good line of sight conditions. The $5.9 \mathrm{GHz}$ antennas mounted on the roof of the vehicle were connected to the communication devices by $4 m$ long RF cables. Further tests yielded that the quality of the RF cables was degraded, resulting in attenuations beyond $8 \mathrm{~dB}$.

\subsection{MAC Layer Testing}

The data link layer is in charge of direct communication between nodes by means of addressing and control mechanisms. The lower sublayer, the medium access control (MAC) layer, is in charge of assuring a coordinated access of nodes to the medium to avoid simultaneous transmission of packets that would lead to a collision. The ITS-G5 standard, as in 802.11p, uses the CSMA/CA algorithm to avoid collisions on the channel. The data link layer adds a 32 Byte header to the given SDU (service data unit) which contains the source and destination MAC addresses, a BSSID address identifying the access point as well as further fields for protocol identification, quality of service (QoS), etc. The address fields as well as the mechanism to provide QoS differ from the the IEEE 802.11 standard. This leads to the following set of interoperability test cases on MAC layer:

- BSSID: Due to the absence of an access point, the stations should operated outside the context of a BSS (Basic Service Set) and the BSSID address inside the MAC header should have all bits set to 1 . Configuring the radio interface to work in monitor mode makes it possible to visualize the complete 32 Byte MAC header and check that this value is correctly set.

- Destination MAC address: According to the standard, ITS stations should support unicast and broadcast communication. The distinction is done using the destination address field in the MAC header, which in broadcast mode should have all bits set to one.

- Source MAC address: ITS stations should continuously monitor whether a neighboring station is using the same MAC address and in this case generate a new address to resolve the conflict.

- QoS: As stations work outside the context of a BSS, QoS for ITS is provided on the basis of EDCA (Enhanced Distribution Channel Access) mechanism. Depending to which of four Access Categories (AC) the traffic belongs to, the interframe space length varies, increasing, this way, the priority to access the channel. To verify the correct behavior two devices send continuously (large packets with smallest repetition time) in different access categories while a third device acts as a receiver. At the receiver, packets with higher priority are expected to exhibit a smaller PER than the ones with lower priority to PASS the test.

The results of executing these test cases on the given $\mathrm{C} 2 \mathrm{X}$ prototypes under test is summarized in table 2 
Table 2. Test results for MAC layer interoperability tests

\begin{tabular}{|l||c|c|c|}
\hline Test Case & Radio A & Radio B & radio C \\
\hline BSSID & PASS & PASS & PASS \\
Destination MAC address & PASS & PASS & PASS \\
Source MAC address & PASS & PASS & PASS \\
QoS & PASS & N $/$ A & N/A \\
\hline
\end{tabular}

\subsection{Network Layer Testing}

In fig. 1 the networking layer can be identified above IEEE802.11p layer. Adhoc networks formed by $\mathrm{C} 2 \mathrm{X}$ communication systems need a special routing algorithm, which is based on geographic addressing and should be able to forward data towards nodes within a destination area. GeoNet is a set of networking protocols developed within a European project [5] and that is likely of being the future networking layer for $\mathrm{C} 2 \mathrm{X}$ communication. Four different geographical routing schemes are defined by GeoNet: geographic unicast, geographic anycast, geographic broadcast and topological broadcast. This work puts its focus on geographical and topological broadcast, as these schemes are the most relevant in current $\mathrm{C} 2 \mathrm{X}$ communication.

Geographical broadcast (GeoBroadcast) delivers data over multiple hops until reaching all the nodes inside a given destination area. The forwarding algorithm behind the GeoBroadcast scheme is Greedy Perimeter Stateless Routing (GPSR) 4. GPSR takes a decision using information about immediate neighbors and chooses as next forwarder the closest node to the destination area. This way geographical broadcast is suited for transmitting, for example, decentralized environmental notification (DEN) 12 messages that inform about events in a certain geographically delimited area. Figure 3 shows the GeoBroadcast process schematically.

On the other hand, topological broadcast (TopoBroadcast) forwards data to all nodes located within a specific number of hops. This scheme is suited for transmitting, for example, cooperative awareness messages (CAM) [1], a beacon with state information sent periodically by each $\mathrm{C} 2 \mathrm{X}$ participant.

Two implementations by Hitachi and NEC of this algorithm have been used to test their interoperability. The following test cases have been identified:

- Tx and Rx of networking packets in a point-to-point scenario: The aim is to verify the ability of communication units to exchange networking packets directly. After verifying that devices can both send and receive network packets, it is possible to test further features in more complex scenarios. For this, two communication devices are placed in range of each other. One of the devices generates first a topological broadcast message with hop limit of one and then geographical broadcast message with a circular destination area that includes both devices. Both messages should be received at the receiving counterpart. 
- Store and forward capability: The store and forward buffer is a component of the networking layer, which is used to store networking packets when no neighboring node is in range. When a new neighbor appears, the stored packets are forwarded to it. This test case checks if this functionality is performed correctly at each of the nodes using two communication devices. The sender generates a GeoBroadcast packet, while the receiver device has its networking layer switched off. When the networking layer of the second unit is switched on, it is possible to check if the sender device forwarded the stored packet correctly.

- GeoBroadcast packet forwarding: This test case checks the capability of a node to forward packets. Therefore, three communication units are needed to perform this test. One of them is the sender and the test will check if only the node closest to the destination area receives a forwarded packet.

- TopoBroadcast packet forwarding:Testing TopoBroadcast forwarding is performed with three devices, one in range of the next one, verifying that the central node decreases the hop counter when forwarding a packet and that the last receiver does not forward the packet as the counter reaches zero.

- Flooding inside the destination area 1: The next step is to check the broadcast process when forwarding packets inside the destination area. According to [5] nodes inside the destination area should forward a packet just once. Three communication units are needed to conduct this test case. When the three units are inside the destination area, it has to be checked that they just forward the packets once whereas when locating one unit outside the destination area, it has to be verified how the unit receives but does not forward the packets.

- Flooding inside the destination area 2: Further on, a node located outside the destination area should drop packets which reaches him from inside the area.

Table 3 summarizes the results of the four test cases performed on both available implementations $\mathrm{A}$ and $\mathrm{B}$.

Table 3. Test results for networking layer interoperability tests

\begin{tabular}{|l||c|c|}
\hline Test Case & Implementation A & Implementation B \\
\hline Direct Communication & PASS & PASS \\
Store \& Forward & PASS & FAIL \\
GB forwarding & PASS & PASS \\
TB forwarding & PASS & PASS \\
Flooding 1 & PASS & PASS \\
Flooding 2 & PASS & PASS \\
\hline
\end{tabular}




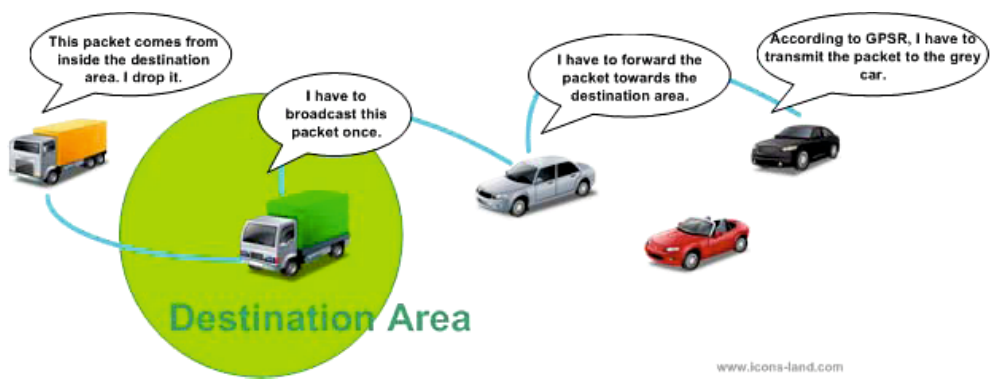

Fig. 3. GeoBroadcast Process

Direct node to node communication by means of topological broadcast messages is performed correctly with both implementations. When using GeoBroadcast communication, only one of the implementations correctly forwarded a previously stored message to a new neighbor. In principle both networking layers implement correctly the GPSR algorithm as described in [5]. Nevertheless, the third test case revealed a slight discrepancy with the specification. The generating node always sent the packet to all surrounding neighbors, instead of selecting the nearest node to the destination area or, in the absence of neighbors, storing the message in the store\&forward buffer. This fact does not impede networking interoperability, but increases the load on the channel by creating several competing routes towards the destination area. Once the GeoBroadcast packet reaches the destination area, every node correctly forwards the packet once and discards packets coming form inside the area. The proposed networking layer test cases showed, that both implementations under test were fundamentally correct, but showed small flaws, that should be revised in further versions.

\subsection{Application Layer Testing}

Interoperability testing on application layer is somehow abstract. The large list of C2X use cases defined by the ETSI in its Basic Set of Applications [10] and their corresponding set of functional requirements, can be seen as a basis for conformance testing, where each of the requirements is checked for its rightness using a single unit and generating artificially the appropriate input. Further on, the correctness and completeness of the exchanged CAM, DENM and GLOSA messages according to their respective specifications, as well as timing requirements for CAM messages, are considered part of previous conformance testing.

Moving towards interoperability testing, the aim is verifying the right behavior of $\mathrm{C} 2 \mathrm{X}$ use cases during their execution on the field involving several participants. Thus, the aim is to check if vehicles receive and show corresponding warnings when they are relevant for drivers. This layer is divided in two sublayers: the use-case sublayer and the facility sublayer. The facility sublayer offers services to use-cases such as positioning or relevance checking. Usually, use-cases receive messages from other vehicles, verify the relevance of the messages and warn the driver if necessary. 
The C2X implementation developed in PreDrive C2X and used in the scope of this work, featured the following use cases:

- Road Works Warning (RWW)

- Emergency Electronic Brake Light (EEBL)

- Car Brakedown Warning - CBW

- Green Light Optimal Speed Advisory (GLOSA)

- In-vehicle Signage (IVS)

RWW and IVS are use cases, where infrastructure components send DEN messages periodically to the surrounding vehicles, whereas EEBL and CBW are use cases where the DEN messages are generated at a vehicle. While the former involve testing the receiving end, as the sender just repeats predefined messages, the latter require to include the sender and the receiver in the test case, as some kind of condition has to be fulfilled at the sender for starting generating DEN messages. In GLOSA, on the other hand, an infrastructure component sends traffic light status messages periodically to the approaching vehicles.

Although each use case is different in its purpose, there are similarities in their operation. Next, a list of test cases for these $\mathrm{C} 2 \mathrm{X}$ applications according to [10] is presented:

- Test 1: DENM generation. The test checks if, according to the input sensors (inertial measurement data and brake status for EEBL or speed and emergency lights status for CBW), a DEN message is generated correctly inside the vehicle.

- Test 2: Relevance checking. This test verifies if an incoming DEN message is relevant for a driver. Position, speed and heading of the vehicle, distance to the event and radius are taken into consideration to evaluate the relevance.

- Test 3: Displaying of warning to the driver. This test checks whether a relevant warning with highest priority in the warning queue was actually forwarded to the driver's Human Machine Interface (HMI).

- Test 4: Revoking of warnings. This test verifies that an expired or not relevant warning is removed from the driver's HMI.

- Test 5: Overall delay. This test measures the delay since a DEN message is generated until the actual warning is displayed on the receiving end. Depending on the use case, the delay shall be as small as $100 \mathrm{~ms}$.

CODAR Viewer [6], a tool created by the DLR to monitor and visualize C2X test sites, was further developed to control field operational tests and extract test data out of the vehicles. This data is sent towards a server, where the 
Table 4. Test results for C2X use-case interoperability tests

\begin{tabular}{|l||c|c|c|c|c|}
\hline Test Case & App 1 & App 2 & App 3 & App 4 & App 5 \\
\hline Test 1 & N/A & PASS & PASS & N/A & N/A \\
Test 2 & FAIL & PASS & PASS & PASS & PASS \\
Test 3 & PASS & PASS & PASS & PASS & FAIL \\
Test 4 & PASS & PASS & PASS & PASS & FAIL \\
Test 5 & PASS & PASS & PASS & PASS & PASS \\
\hline
\end{tabular}

running use-case is monitored and evaluated online by a tester. The proposed test cases were performed on the given $\mathrm{C} 2 \mathrm{X}$ applications under test. Table 4 gives exemplary results for these test cases, which helped to detect failures during the implementation phase.

During the implementation phase, one of the applications showed problems with the correct displaying of warnings and with their revoking. Another application had too stringent conditions for accepting a warning as relevant with the consequence that no HMI alert was given to the driver. These issues were later solved correctly.

\section{Conclusions and Future Work}

This paper has presented a variety of interoperability test cases to be performed on different communication layers on European ITS systems. Starting on the physical layer direct ITS-G5 radio-to-radio communication has been tested, being frequency channel, bit rate or bandwidth the most common accessible parameters. One layer above, test cases for the MAC layer have been explained. Both, physical and MAC layer test cases have been applied on three different $\mathrm{C} 2 \mathrm{X}$ communication devices from different manufacturers. On networking layer, the focus has been put on GeoNet specification, as the most likely set of algorithms to be standardized by the ETSI for ITS systems. A set of test cases for geographic and topological broadcast have been presented along with the results on their execution on two prototypical implementations. On application layer, five C2X use cases developed within the European project PreDrive C2X have been evaluated using the CODAR Viewer tool.

Additionally, interoperability testing on two further aspects of C2X systems is considered by the authors to be essential and to require exhaustive analysis. Both, security that deals with integrity, authenticity and trustworthiness in C2X communication to avoid malicious usage of the system, and privacy protection to preserve anonymity or to prevent recording of movement, will also require extensive interoperability testing due to the involvement of different entities (signer, verifier, certificate authority, etc.). Further on, another ITS architecture implementation, namely the one developed in the European project CVIS [7, should be analyzed for interoperability in future work. This architecture follows the COMeSafety definition and is based on ISO CALM. In spite of large similarities 
on application, facility and lower communication layers with the implementation analyzed in this work, CALM is strongly focused on IPv6 as networking strategy.

\section{Acknowledgment}

The work for this paper was accomplished in the frame of the European project ITS TestBeds. The ITS prototypes used during this work were developed in the European project PreDrive C2X.

\section{References}

1. Baldessari, R., Boedekker, B., Deegener, M., Festag, A., Franz, W., Christopher Kellum, C., Kosch, T., Kovacs, A., Lenardi, M., Menig, C., Peichl, T., Roeckl, M., Seeberger, D., Strassberger, M., Stratil, H., Voegel, H.-J., Weyl, B., Zhang, W.: Car-2-car communication consortium - manifesto, 08 (2007)

2. Bossom, R., Brignolo, R., Ernst, T., Evensen, K., Frötscher, A., Höfs, W., Jääskeläinen, J., Jeftic, Z., Kompfner, P., Kosch, T., Kulp, I., Kung, A., Mokaddem, A.-K., Schalk, A., Uhlemann, E., Wewetzer, C.: European ITS communication architecture - Overall framework - Proof of concept implementation. Technical report, EC Information Society Technologies Programme (February 2010)

3. ETSI Technical Comitee Methods for Testing and Specification. Methods for testing and specification (mts); internet protocol testing (ipt); generic approach to interoperability testing. Technical report, ETSI, ETSI Guide ETSI ES 302571 (Septmeber 2008)

4. Karp, B., Kung, H.T.: GPSR: greedy perimeter stateless routing for wireless networks, pp. 243-254 (2000)

5. Kovacs, A.: GeoNet Strep N216269 D2.2 Final GeoNet Specification (January 2010)

6. Kranz, M., Franz, A., Röckl, M., Andreas, L., Strang, T.: Codar viewer - a situation-aware driver assistance system. In: Advances in Pervasive Computing Pervasive 2008 Demonstration, vol. 236, pp. 126-129. Austrian Computer Society (2008)

7. Solberg, A., Schmid, A., Baggen, M., Alesiani, F., Stratil, H., Bossom, R., Forkert, S., Schlingelhof, M., Burkert, A., Haverkamp, S., Mathias, P., Jeftic, Z., Evensen, K., Olsen, E., Fischer, H.-J., Fazekas, I., Gaillet, J.-F.: D.CVIS.3.4 - Final Architecture and System Specifications (June 2010)

8. ETSI Technical Comitee Intelligent Transport Systems. Intelligent transport systems (its); radiocommunications equipment operating in the $5855 \mathrm{mhz}$ to 5925 mhz frequency band; harmonized en convering the essential requirements of article 3.2 of the r\&tte directive. Technical report, ETSI, ETSI Guide ETSI ES 202237 (April 2007)

9. ETSI Technical Comitee Intelligent Transport Systems. European Profile Standard for the Physical and Medium Access Control Layer of Intelligent Transport Systems Operating in the $5 \mathrm{GHz}$ Frequency Band. Technical report, ETSI, ETSI Standard ETSI ES 202663 (November 2009) 
10. ETSI Technical Comitee Intelligent Transport Systems. Intelligent Transport Systems (ITS); Vehicular Communications; Basic Set of Applications; Part 1: Functional Requirements. Technical report, ETSI, Technical Specification ETSI TS 102637-1 (September 2010)

11. ETSI Technical Comitee Intelligent Transport Systems. Intelligent Transport Systems (ITS); Vehicular Communications; Basic Set of Applications; Part 2: Specification of Cooperative Awareness Basic Service. Technical report, ETSI, Technical Specification ETSI TS 102637-2 (April 2010)

12. ETSI Technical Comitee Intelligent Transport Systems. Intelligent Transport Systems (ITS); Vehicular Communications; Basic Set of Applications; Part 3: Specifications of Decentralized Environmental Notification Basic Service. Technical report, ETSI, Technical Specification ETSI TS 102637-3 (September 2010) 